\title{
Ball Mill Shell Vibration Signal Analysis Strategy Based on DEM-FEM Method and Multi-Component Signal Adaptive Decomposition Technique
}

\author{
Jian Tang ${ }^{1,2, a}$, Zhiwei $\mathrm{Wu}^{2, \mathrm{~b}}$ and Zhuo $\mathrm{Liu}^{2, \mathrm{c}}$ \\ ${ }^{1}$ Research Institute of Computing Technology, Northern Jiaotong University, Beijing, China \\ ${ }^{2}$ State Key Laboratory of Synthetical Automation for Process Industries, Northeastern University, \\ Shenyang, China \\ apowernature@126.com, bwuzhiwei_03@126.com, ${ }^{\mathrm{c}}$ liuzhuo@mail.neu.edu.cn
}

Keywords: DEM, FEM, Ball mill, Vibration signal analysis, EMD, HVD.

Abstract. Ball mills are heavy rotating mechanical devices of industrial processes. Ball mill shell vibration signal has characteristics of multi-component and non-stationary. It has been a new focus to estimate some difficult-to-measure process parameters, such as load parameters within mill. The detailed analysis of the shell vibration signal production mechanism can help us construct estimation model with reasonable physical interpretation and well generalization performance. Thus, a new strategy of the shell vibration signal analysis based on DEM-FEM method and multi-component signal adaptive decomposition technique is proposed. At first, a simple ball mill shell FEM model is constructed. Then, this model is imported to DEM for producing impaction forces to mill shell. Then, these forces are added on the FEM model to active the mill shell vibration for producing the virtual accelerator vibration signal. Finally, the virtual shell vibration signal is analyzed with the multi-component signal adaptive decomposition techniques, such as empirical mode decomposition (EMD) and Hilbert vibration decomposition (HVD). Further research would focus on experimental calibration to give reasonable simulation results.

\section{Introduction}

Ball mills are used widely in the chemical and mineral industry processes for size reduction. They are heavy and highly energy consumptive devices. Load parameters within ball mill relate to production quality and quantity of these industrial processes. Ball mills' mechanical and vibration signals are used to measure these difficulty-to-measure process parameters. Due to development of the vibration measurement instruments and improvement of the wireless communication, the reliable measurement of the ball mill shell vibration signals has been realized. As the mill shell vibration signal is more sensitivity than the other signals, it has been a new focus to model mill load parameters [1]. However, most of the soft sensor models are constructed only from the viewpoint of data-driven method. It is very necessary to give detailed physical interpretations based the vibration production mechanism and to understand the ball mill grinding process deeply.

Theoretically, there are millions balls inside the mill, which are layered. The different layers of balls impact on the material and the mill shell are difference in terms of amplitudes and periods. Thus, a strong shell vibration signal is mixed with multiple sub-signals at various time scales. Some signal adaptive decomposition techniques, such as empirical mode decomposition (EMD) [2], ensemble EMD (EEMD) [3] and Hilbert vibration decomposition (HVD) [4], are used to pre-process the shell vibration signal before construct soft sensor model. Many sub-signals with different physical interpretations in theory are obtained. However, only a few ones can be explained based on prior knowledge. Thus, it is very necessary to find a new approach to understand these sub-signals clearly.

Mathematical simulation studies of the mill grinding process are used widely for optimized structure parameters design [5,6]. Discrete element method (DEM) are always used to analysis the balls' motion. Finite element method (FEM) are always used to analyze ball mill shell and the inside lines. Therefore, a numerical description of mill systems by combining DEM and FEM model was 
presented [7]. However, all the objectives of the above researches are not for the construction of soft sensor model.

Motivated by the above problems, we proposed a new strategy of ball mill shell vibration signal analysis based on DEM-FEM method and multi-component signal adaptive decomposition techniques. It maybe helps us to interpretation the constructed soft sensor model deeply.

\section{Ball Mill Shell Vibration System Analysis}

Normally, there are millions of balls inside the mill. Theoretically, these balls arrange in different layers. They impact the mill shell direct or indirect with different amplitudes and periods. Thus, the mill shell vibration signal caused by these forces must be consist of sub-signals with different time scales. This production process can be desribed with the Fig. 1.

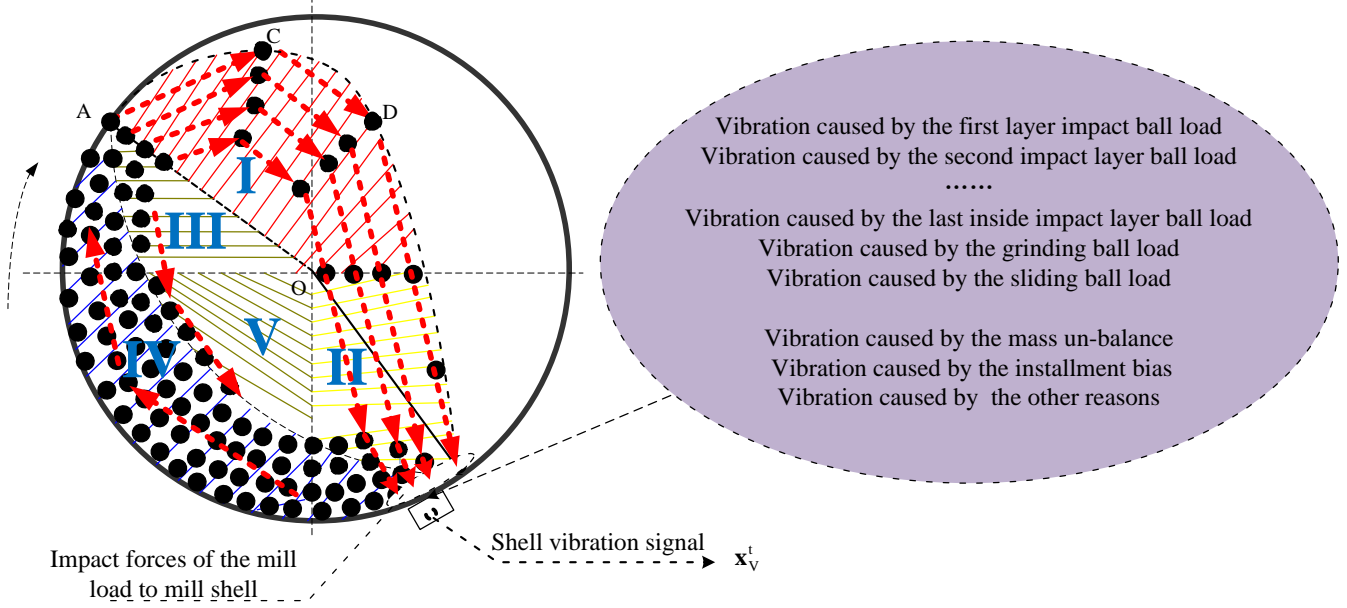

Fig. 1. Production diagram of the shell vibration and acoustic signals

Moreover, mass un-balance and installment bias of the ball mill, and other reasons can also stir up the shell vibration. Therefore, the shell vibration signal has characteristics of multi-component and multi-scale.

\section{Multi-Component Signal Adaptive Decomposition Techniques}

The original EMD decomposition algorithm has been applied widely in the last decade. Modified EMD algorithms, such as ensemble EMD (EEMD), is also used successfully in different background. More recently, one of the authors proposed HVD. These algorithms can decompose multi-component non-stationary signal into sub-signals with different interesting information.

The EMD decompose original signal automatically to a serial of intrinsic mode functions (IMFs). It is based on iteration process with the following steps: (1)Estimation of all local extremas; (2)Spline fitting of all local minimas and maximas, ending up with two extremas functions as the upper and the lower envelopes; (3)Calculation of the average function between the upper and the lower envelopes; (4)Extraction of the average from the initial signal; (5)Iteration on the residual signal.

The mode-mixing phenomenon of EMD is defined as: (a) an IMF containing signals of widely disparate scales; (b) signals of similar scale residing in different IMF components. EEMD was proposed to overcome this promblem. The EEMD algorithm is also based on an iteration with the following steps: (1)Initialize the number of trials in the ensemble number $\mathrm{M}$ and the amplitude of the added white noise; (2)Add white noise to original signal and decompose the new signals with EMD; (3)Perform M times"steps" (2); (4)Calculate the ensemble mean values.

The HVD method is based on the Hilbert transform (HT) presentation of the instantaneous frequency (IF) and does not involve spline fitting and empirical algorithms. The decomposition is based on the following assumptions: (a) The underlying signal is formed by a superposition of symmetric quasi-harmonic functions; (b) The envelopes of each oscillating component differ from 
each other; (c) The total length of each component spans several periods of the corresponding slowest component. This method is also an iterative process, and every iteration step includes the following procedures: (1)Estimate the IF of the largest component; (2)Detect the corresponding envelope of the largest component; (3)Subtract the largest component from the original composition; (4)Iteration on the residual signal.

Some adative signal decomposition algortihms, such as EMD and HVD, have been used to decompose the shell vibration signal into different sub-signals. However, there are only a few sub-signals can be explained detailed based on the prior knowledge.

\section{DEM-FEM- based Mathematical Simulation}

Simulation of the ball motion with the ball mill may be can help us to understand the impact forces of the balls to the mill shell. The discrete element method (DEM) can simulate the dynamics of particle processes using the computational method. In numerical algorithm, every disc has properties of radius, mass, moment of inertia, and collision. Each disc is identified separately in the assembly with this attributes. Then, Newton's second lawn is used to describe the motion of discs; the discs in collision are described with a force displacement model. It has been successfully applied to grinding mills [8]. The particle collision information can be extracted from the DEM simulation process. Normally, the impact energy and force distributions are used to calculate the grinding rate in mills in most of the studies; Some authors studied also the inter-particle force law and contact parameters in DEM simulations. The DEM model gives the detailed information on charge motion, collision forces, energy loss spectra, power consumption [9]. Thus, it is important for gaining a deeper understanding of the grinding process itself. Therefore, by using the DEM method, we can give the detailed explanation of the different forces to active the vibration of the mill shell.

Thus, after these different forces are obtained, the next problem is how to model mill shell vibration response. Finite element method (FEM) has been used to mechanical structural analysis widely. It is a numerical solution method based on continuum mechanics modeling. In mathematics, FEM is a numerical technique for finding approximate solutions to boundary value problems for partial differential equations. It uses subdivision of a whole problem domain into simpler parts, called finite elements, and variational methods from the calculus of variations to solve the problem by minimizing an associated error function.

The DEM-FEM can be used to study the structural response and its influence on the balls motion. Therefore, it is always used to optimize the material selection of the mill structure. Moreover, the DEM-FEM models give a direct coupling between force, stress and deflection for the whole mill shell system than that of the DEM model.

\section{The Proposed Ball Mill Shell Vibration Analysis Strategy}

Based on the above analysis, a new strategy is proposed for the detailed interpretation of the multi-component of the shell vibration signal. It is shown as in Fig.2.

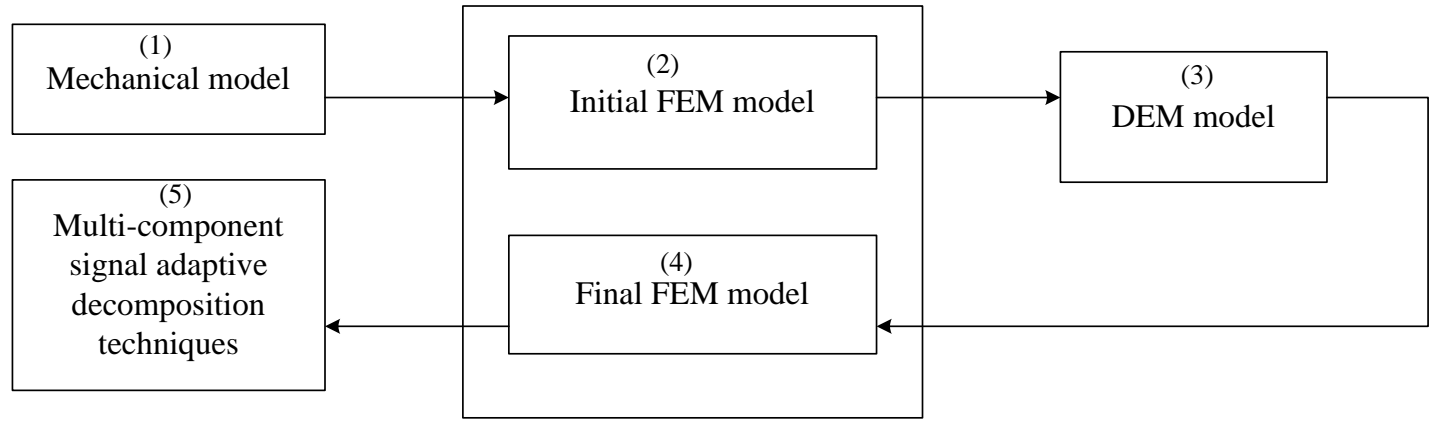

Fig. 2. The proposed ball mill shell vibration signal anaysis strategy 
Fig. 2 shows that there are total four software using in the proposed strategy. The first one is the mechanical modeling software to construct mechanical model of the ball mill shell vibration system, such as UG. The second one is the FEM software to produce the virtual shell vibration signal under different impaction $t$ forces of the steel balls, such as ABAQUS. The third one is the DEM software to produce impact forces of different steels to mill shell, such as EDEM. The last one is the software to docompose the virtual shell vibration singal into different sub-signals, such as EMD or HVD.

There are five steps to implement the proposed stratedy:

Step(1): Using UG to construct mechanical model of the ball mill shell;

Step(2): Import the UG model into ABAQUS to construct the initial FEM model and export FEM model for EDEM to simulate impact forces;

Step(3): Import the initial FEM model into EDEM and configure ball load insided ball mill, then run this DEM model to obtain different impact forces to the mill shell and export the simulation results to the former built FEM model;

Step(4): Import the DEM model's results into ABAQUS to obtain the final FEM model, and then obtain the virtual vibration signal of the mill shell mechanical system;

Step(5): Decompose the virtual vibration signal into different sub-signals.

Given different ball load and differet simulaiton parameters, the diffrent sub-signals can be obtained. Thus, by detailed experimental design, interprestations of differetn sub-signals may be obtained. Then, the results can give a assistant to understand the measured shell vibration signasl. By experiments under different grinding constitions, such as water mill, dry mill and wet mill, some theoritcal results can assist to construct effective soft sensor model of mill load parameters.

\section{Conclusions and discussions}

Aim to construct soft sensor model with reasonable physical interpretation and well generalization performance, a new strategy of ball mill shell vibration signal analysis is proposed. It needs commercial software to implement this process. Theoretically, it can be realized. However, there are several questions should be considered in its implementation: (1) How to integrate different software seamlessly? (2) How to decrease computing consumption? (3) How to calibrate different simulation parameters in DEM model? (4) How to select different analysis parameters in different software in terms of global optimization?

\section{Acknowledgements}

This work is partially supported by the post doctoral National Natural Science Foundation of China (2013M532118, 2015T81082), National Natural Science Foundation of China (61573364, 61273177, 61503066), China National 863 Projects (2015AA043802).

\section{References}

[1] J. Tang, T.Y. Chai, W. Yu and L.J. Zhao: IEEE Transactions on Automation Science and Engineering Vol.10 (2013), p. 726.

[2] N.E. Huang: Proceedings of the Royal Society of London A Mathematical Physical \& Engineering Sciences Vol.454 (1998), p. 903.

[3] Z.H. Wu, N.E. Advances in Adaptive Data Analysis Vol.55, (2009), p. 193.

[4] M. Feldman: Journal of Sound and Vibration Vol.295 (2006), p. 518.

[5] B.K. Mishra: Int. J. Miner. Process Vol.71 (2003), p. 73.

[6] B.K. Mishra: Int. J. Miner. Process Vol.71 (2003), p.95. 
[7] P. Jonsén, B.I. Pålsson and K. Tano: Minerals Engineering Vol.24 (2011), p.236

[8] B.K. Mishra and R.K. Rajamani: Applied Mathematical Modelling Vol.16 (1992), p.598.

[9] K.T. Tano, B.I. Pålsson and A. Sellgren: Miner. Eng Vol.22, (2005), p.710. 